\title{
Measurement of transversity signals in two hadron production at COMPASS
}

\author{
Frank Massmann \\ on behalf of the COMPASS-collaboration \\ Helmholtz-Institut fuer Strahlen- und Kernphysik \\ University of Bonn \\ E-Mail: massmann@iskp.uni-bonn.de
}

\begin{abstract}
The quark structure of the nucleon at the twist two level can be completely described with three quark distribution functions: the spin averaged distribution function $q(x)$, the helicity distribution $\Delta q(x)$ and the transverse spin distribution $\Delta_{T} q(x)$. This last function, referred to as transversity, is chiral-odd and can only be measured in combination with another chiral-odd function.

COMPASS is a fixed target experiment at the CERN SPS M2 beamline. Its target $\left({ }^{6} \mathrm{LiD}\right)$ can be both longitudinally and transversely polarized with respect to the polarized $160 \mathrm{GeV} / \mathrm{c} \mu^{+}$-beam. In transverse configuration $\Delta_{T} q(x)$ can be measured in semi-inclusive deep-inelastic scattering (SIDIS), requiring the detection of hadronic products. A rather new probe of the transverse spin distribution function $\Delta_{T} q(x)$ is the measurement of two hadron production, introducing the chiral odd interference fragmentation function $H_{1}^{\varangle}\left(z, M_{h}^{2}\right)$. Results are presented for the years 2003-2004 including particle identification by using the information of the RICH detector. The analysis was performed using all positive/negative charged pairs and $z$-ordered pairs.
\end{abstract}

Neglecting any intrinsic quark transverse momenta $\vec{k}_{T}$ there are three parton distribution functions (PDF) completely describing the contribution of quarks to the spin structure of the nucleon: the unpolarized or averaged quark distribution function $q(x)$, defined as $q(x)=q^{\uparrow}(x)+q^{\downarrow}(x)$, where $q^{\uparrow}(x)$ is the quark distribution function of the quarks polarized parallel to the nucleon spin and $q^{\downarrow}(x)$ the quark distribution function of the quarks polarized antiparallel to it. The helicity-distribution $\Delta q(x)$, defined as $\Delta q(x)=q^{\uparrow}(x)-q^{\downarrow}(x)$ in a longitudinally polarized nucleon and finally the transversity distribution, which is also defined as $\Delta_{T} q(x)=q^{\uparrow}(x)-q^{\downarrow}(x)$ but in a transversely polarized nucleon.

Naively one would expect the transversity distribution to be the same as the helicity distribution, but the breakup of the rotational invariance at relativistic energies has to be taken into account. Whereas the averaged PDF $q(x)$ and the helicity distribution $\Delta q(x)$ can be measured in inclusive deep inelastic scattering (DIS), this is not possible for the transversity distribution $\Delta_{T} q(x)$ due to its chiral odd nature. As a consequence the spin of the struck quark flips. One possibility to conserve the total angular momentum of the whole scattering process is the production of hadrons carrying the difference of angular momentum of the struck quark before and after the scattering. The production of hadrons from the struck quark is described by fragmentation functions (FF) which for transversely polarized quarks contains a chiral odd part. Therefore in semi inclusive DIS the transversity distribution is accessible via the convolution of transversity distribution and FF. Up to now most attention was turned to measurements of single hadron production in semi inclusive DIS, where the convolution of the transversity distribution function and the Collins FF $\Delta_{T}^{0} D_{q}^{h}\left(z, p_{T}^{h}\right)[1][2]$ is measured. These measurements led to first interpretations of transversity-distribution[3].

An alternative access to transversity is the measurement of the azimuthal asymmetry in the inclusive production of hadrons pairs. This asymmetry is related to the product of the transversity distribution and 
the chiral odd "interference" FF $H_{1}^{\varangle}\left(z, M_{h}^{2}\right)$, which is the spin dependent part of the FF for a transversely polarized quark into two unpolarized hadrons. The term "interference" refers to the property, that the production of the pair is due to an interference between different wave states of the hadron pairs. The FF $H_{1}^{\varangle}\left(z, M_{h}^{2}\right)$ can be disentangled in a way that there are two different FF:

$$
H_{1}^{\varangle}\left(z, M_{h}^{2}\right)=H_{1}^{\varangle, s p}\left(z, M_{h}^{2}\right)+\cos \theta H_{1}^{\varangle, p p}\left(z, M_{h}^{2}\right),
$$

the first one represents the interference between $\mathrm{s}^{-}$and $\mathrm{p}$-wave states of the pairs, whereas the second one is related to the interference between $\mathrm{p}$-wave states only. $\theta$ is the angle between $\vec{P}_{1}$, which is the momentum of $h_{1}$, and the sum vector of the momenta of the two hadrons $\vec{P}_{h}$ in their cms-frame (see Fig.1 (right)). The properties of "interference" FF are studied in more detail in $[4,5,6,7,8,9,10,11]$.

At leading twist the FF of an unpolarized quark into unpolarized hadrons $D_{q}^{2 h}\left(z, M_{h}^{2}\right)$ has to be added to the "interference" $\mathrm{FF} H_{1}^{\varangle}\left(z, M_{h}^{2}\right)$, therefore the FF of a quark q into a pair of two hadrons $h_{1}$ and $h_{2}$ reads:

$$
D_{q}^{2 h}\left(z, M_{h}^{2}\right)+H_{1}^{\varangle}\left(z, M_{h}^{2}\right) \sin \phi_{R S} \sin \theta,
$$

with $\phi_{R S}=\phi_{R}+\phi_{S}-\pi$. $\phi_{R}$ is defined as the azimuthal angle of $\vec{R}_{T}$, which is the transverse component of the vector $\vec{R}=\frac{z_{2} \vec{P}_{1-z_{1}} \vec{P}_{2}}{z_{1}+z_{2}}$, and $\phi_{S}$ is the azimuthal angle of target spin vector. The fraction of the transferred energy carried by one of the two hadrons $h_{1}$ or $h_{2}$ is $z_{1,2}=E\left(h_{1,2}\right) /\left(E_{l}-E_{l \prime}\right)$, with $E_{l}$ being the incoming and $E_{l}$, the scattered lepton energy. $M_{h}^{2}$ is the invariant mass squared of the hadron pair. The angle $\theta$ is explained above. At COMPASS the results are obtained by integrating over $\sin \theta$, where $\theta$ is close to $\pi / 2$ with $<\sin \theta \approx 0.95>$.
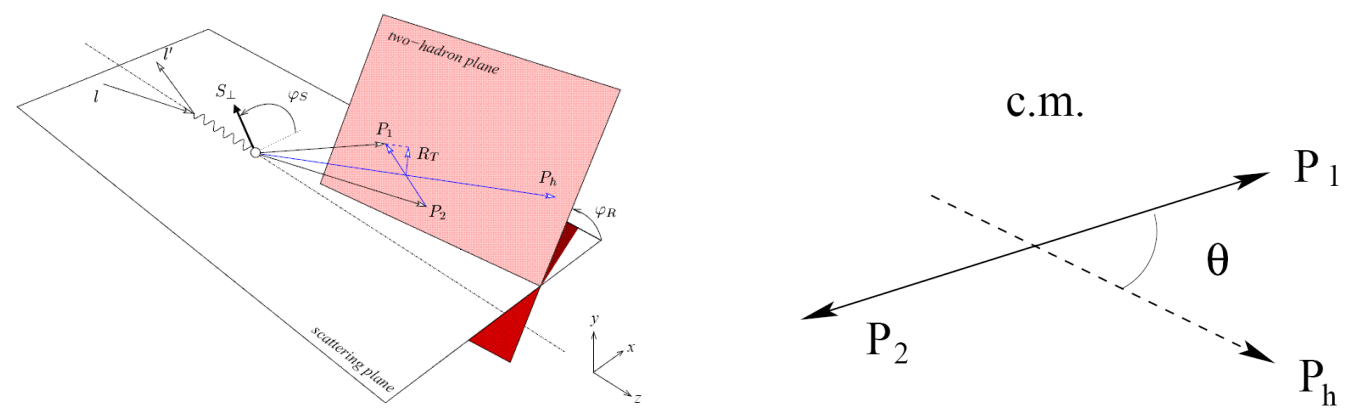

Fig. 1: Description of the angles involved in the measurement of single spin asymmetries in deep inelastic production of two hadrons (left)[11]. The hadron pair in the $\mathrm{cm}$ frame (right); $\theta$ is the $\mathrm{cm}$ polar angle of $P_{1}$ with respect to the direction of $P_{h}$ in the target rest frame.[13]

The measured quantity from which the transversity distribution could be accessed is an asymmetry $A_{\phi_{R S}}$ in the distribution of the azimuthal angle $\phi_{R S}$ :

$$
\frac{\sum_{q} e_{q} \Delta_{T} q(x) H_{1, q}^{\varangle}\left(z, M_{h}^{2}\right)}{\sum_{q} e_{q} q(x) D_{q}^{2 h}\left(z, M_{h}^{2}\right)} \propto A_{\phi_{R S}}=\frac{A_{U T}^{\sin \phi_{R S}}}{D_{N N} f P}
$$

$A_{U T}^{\sin \phi_{R S}}$ is the measured raw asymmetry, which has to be corrected to get the physical asymmetry. These factors are: $f \approx 0.4$ the dilution factor, $P \approx 0.5$ the target polarization and $D_{N N}=(1-y) /\left(1-y+y^{2}\right)$ the kinematical depolarization factor, where $y$ is the fraction of energy transferred to the final hadronic state.

The COMPASS spectrometer[14] receives a $\mu^{+}$-beam of high intensity from the SPS accelerator at CERN. This beam is produced by decaying pions, so the $\mu^{+}$'s are naturally longitudinally polarized at $\approx-0.75$. To make measurements on the spin structure of the nucleon a solid state polarized target of ${ }^{6} \mathrm{LiD}$ was used in the years from 2002-2004. This target could be polarized either in longitudinal or in 
transverse directions with respect to the beam. The target consists of two target-cells, which are polarized in opposite directions. In transverse mode the polarization of both target cells is reversed every week to minimize systematic effects. This method creates two subperiods of data-samples, which are compared in the analysis. About 20\% of data taken in 2002-2004 are taken on a transversely polarized target. For particle identification a RICH detector was used in the years 2003 and 2004. The RICH detector consists of a $\mathrm{C}_{4} \mathrm{~F}_{10}$ gas radiator. In the data analysis on the extraction of the two hadron correlation only events are selected with an incoming beam track having a vertex inside one of the two target cells, which is correlated with a scattered muon track and at least two outgoing hadron tracks. Kinematic cuts on $Q^{2}>1(\mathrm{GeV} / \mathrm{c})^{2}, 0.1<y<0.9$ and $W>5 \mathrm{GeV} / \mathrm{c}^{2}$ were applied to select deep inelastic events above nuclear resonances only and to avoid effects due to radiative corrections and smearing. $Q^{2}$ is the photon virtuality and $W$ the mass of the final hadronic state. To separate hadrons from muons, information of the traversed material was used. Using the RICH information for the year 2003 and 2004, it was possible to identify pions and kaons in the final hadronic sample. Additional cuts on $z>0.1$ for each hadron and $z_{1}+z_{2}<0.9$ were applied to reject the region of target fragmentation and exclusive $\rho$-production. One analysis for two hadron correlation was performed by selecting a data sample of all pairs in an event, with the first hadron having positive charge and the second hadron having a negative charge. Additionally a second analysis used a data sample of only one pair per accepted event, where the first hadron is the hadron with leading $z$ (i.e. maximal energy) and the second is the hadron with the next to leading $z$. Whereas the statistics for this analysis is lower than in the first case, a higher significance of the data sample is expected, looking only at the hadron pairs carrying most information of the scattering process. Considering also the charge and the particle identification, this leads to an amount of 16 data samples (4 charge $(+/+,+/-,-/+$ and $-/-)$ and 4 particle combination $(\pi / \pi, \pi / K, K / \pi$ and $K / K)$ ) for the leading- $z$ analysis. In this analysis events were rejected, if in this event the hadron with the maximum (or second maximum) $z$ was rejected due to other cuts. Information of hadronic calorimeters were used to ensure, that there is no neutral hadron with maximal (or second maximal) $z$.

For the identified two hadron analysis the total amount of accepted all hadron ${ }^{+} /$hadron $^{-}-$pairs in 2003 and 2004 was as follows:

$3.7 \cdot 10^{6} \pi^{+} / \pi^{-}-, 2.4 \cdot 10^{5} \pi^{+} / \mathrm{K}^{-}-, 3.0 \cdot 10^{5} \mathrm{~K}^{+} / \pi^{-}-$and $8.6 \cdot 10^{4} \mathrm{~K}^{+} / \mathrm{K}^{-}-$pairs.

For these data samples the distribution

$$
N\left(\phi_{R S}\right)=\Phi_{a} n \sigma a\left(\phi_{R S}\right)\left(1 \pm A_{U T}^{\sin \phi_{R S}} \sin \phi_{R S}\right)
$$

for each target cell is derived. $\Phi_{a}$ is the muonflux, $\mathrm{n}$ the number of particles in the target, $\sigma$ the spin averaged cross-section and $a\left(\phi_{R S}\right)$ is the unknown product of the angular acceptance and efficiency of the spectrometer. To get rid of the unknown acceptance function, a method called "ratio product method" is used, which is described in detail in [3]. For this method the ratio of the acceptances of the up- and downstream cell stays constant between the two subperiods. Using this method both subperiods and both target cells with opposite polarizations are used by creating the ratio:

$$
F\left(\phi_{R S}\right)=\frac{N_{u p}^{\uparrow}\left(\phi_{R S}\right) N_{d o w n}^{\uparrow}\left(\phi_{R S}\right)}{N_{u p}^{\downarrow}\left(\phi_{R S}\right) N_{d o w n}^{\downarrow}\left(\phi_{R S}\right)},
$$

where up and down refers to one of the two target cells (called upstream and downstream cell) and the arrows indicate the polarization of the corresponding target cell. This ratio is calculated for 16 $\phi_{R S}$-bins and plotted versus $\phi_{R S}$ for one of three variables $x, z$ and $M_{i n v}$, which are also subdivided in certain bins each. These are the three variables the transversity function and the "interference" FF respectively depend on. For each binning in the three variables the $16 \phi_{R S}$-bins are fitted with the function $F\left(\phi_{R S}\right)=p_{0}\left(1+4 A_{U T}^{\sin \phi_{R S}} \sin \phi_{R S}\right)$, with $p_{0}$ being a constant parameter.

The COMPASS results for two hadron correlation from 2002-2004 without particle identification are shown in [15]. Fig.2 shows the results for the years 2003-2004 for all identified $\pi^{+} / \pi^{-}$-pairs (top) and for the $\mathrm{z}$-ordered analysis in the case of all possible $\pi^{+} \pi^{-}$and $\pi^{-} \pi^{+}$combinations (bottom). The results are compatible with zero, which is also the case for the results including kaons, which are not shown here. 

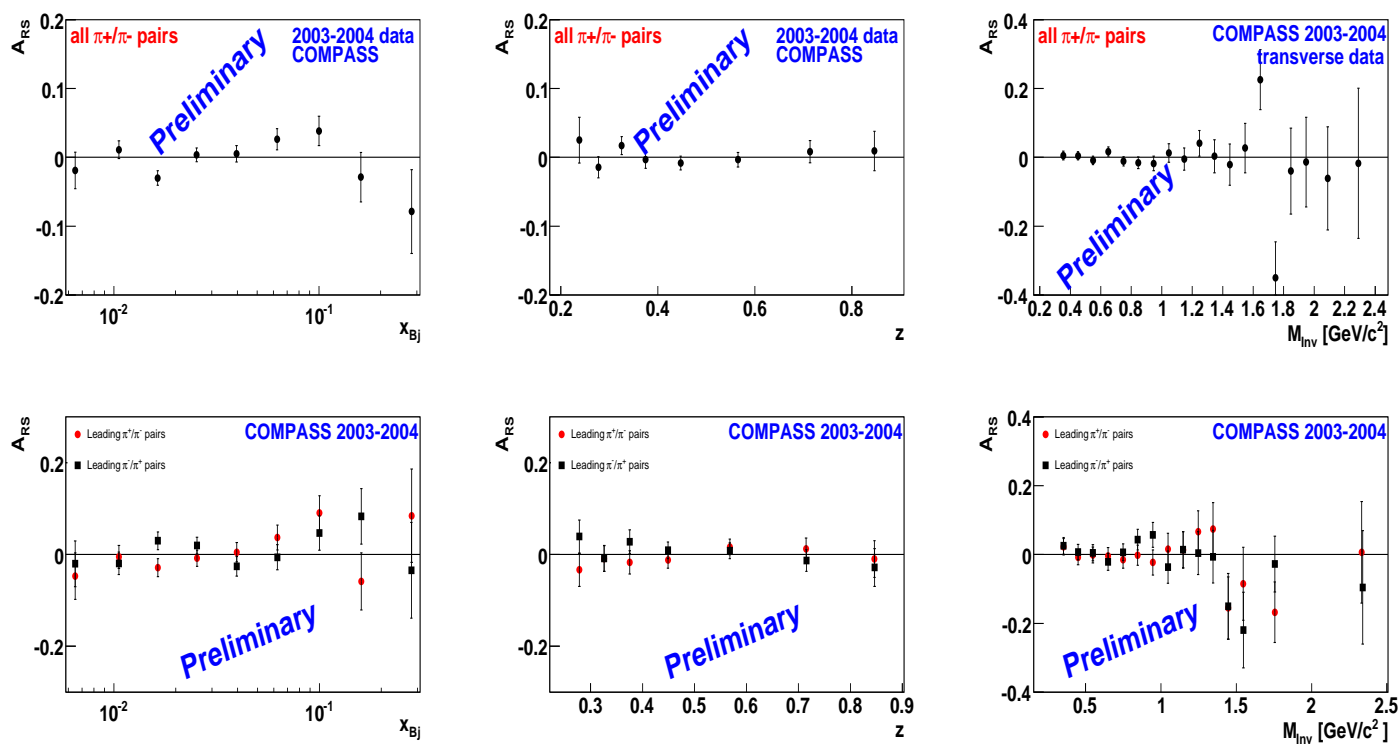

Fig. 2: COMPASS results for 2003-2004 with identified $\pi$. At top: Final asymmetries for all $\pi^{+} / \pi^{-}-$pairs in $x$, $z$ and $M_{i n v}$. At bottom: Final asymmetries for leading $\pi$ analysis with opposite charge in $x, z$ and $M_{i n v}$. The red labels(rhombus) are for leading $\pi^{+} / \pi^{-}$, the black labels(square) are for leading $\pi^{-} / \pi^{+}$.

All results obtained so far for the COMPASS deuteron are thus in agreement with the predictions made in [13], predicting a nearly zero asymmetry for deuteron target in contrast to a proton target, where an asymmetry of measureable size is expected and reported by the HERMES Collaboration. In 2007 COMPASS switched to transversity measurements with a $\mathrm{NH}_{3}$ target to complement the measurements on the deuteron target by a proton target.

\section{References}

[1] V.Yu. Alexakhin et al. [COMPASS Collaboration], Phys. Rev. Lett. 94, 202002 (2005).

[2] Collins, J.1993, Nucl. Phys. B396 161.

[3] The COMPASS-collaboration. A new measurement of the Collins and Sivers asymmetries on a transversely polarised deuteron target. Nucl. Phys. B765, 31-70 (2007). hep-ex/0610068.

[4] J.R. Collins, S.F. Heppelmann and G.A. Ladinsky, Nucl. Phys. B420, 565 (1994).

[5] X. Artru and J. C. Collins, Z. Phys. C69, 277 (1996).

6] R. L. Jaffe, X. Jin and J. Tang, Phys. Rev. Lett. 80, 1166 (1998).

[7] M. Radici, R. Jakob and A. Bianconi, Phys. Rev. D65 074031 (2002).

[8] A. Bacchetta and M. Radici, hep-ph/0608037.

[9] A. Bianconi, S. Boffi, R. Jakob and M. Radici, Phys. Rev. D62, 034008 (2000).

[10] A. Bacchetta and M. Radici, Phys. Rev.D67, 094002 (2003).

[11] A. Bacchetta and M. Radici, Phys. Rev. D69, 074026 (2004).

[12] X. Artru, hep-ph/0207309 (2002).

[13] A. Bacchetta and M. Radici. Modeling dihadron fragmentation functions, hep-ph/0608037.

[14] The COMPASS-collaboration. The COMPASS Experiment at CERN. hep-ex/0703049, accepted by NIM A.

[15] R.Joosten on behalf of the COMPASS-collaboration. Transversity signals in two hadron correlation at COMPASS. Proceedings for 17th International Spin Physics Symposium (SPIN06). 